\section{PRESCRIPCIONES TÉCNICAS PARA VIVIENDAS E INFRAESTRUCTURAS CRÍTICAS EN INTERFAZ URBANO- FORESTAL FRENTE A INCENDIOS: EL CASO DE SAN JOSÉ DE MAIPO, CHILE}

Miguel Castillo ${ }^{1 *}$, Rose Marie Garay ${ }^{2,4}$ y Ricardo Tapia ${ }^{3,4}$

\section{RESUMEN}

Se analizó la condición de vulnerabilidad de edificaciones frente al impacto de los incendios forestales en áreas de interfaz localizadas en San José de Maipo, Región Metropolitana, Chile. Para este propósito recopilamos datos de población, estadísticas de incendios, normas de construcción, legislación vigente y en general una extensa red de información desde el año 2000 en adelante. Con estos antecedentes evaluamos las áreas con mayor presencia de viviendas en bosques y las principales normas de construcción vigentes, como también las características de resguardo frente al fuego para distintas categorías de construcción: viviendas, colegios, hospitales, y en general todo tipo de infraestructura crítica que cumple funciones de defensa y protección frente a catástrofes. Los resultados muestran una alta exposición de prácticamente todas las categorías de edificaciones, debido a que se localizan en áreas de máxima vulnerabilidad. Respecto a las construcciones existentes, se realizan propuestas para mejorar el entorno inmediato a las áreas con mayor vulnerabilidad. Se trata de una experiencia piloto en Chile, basada en los resultados del Proyecto FONDEF it16i10003 (Chile), sobre protección y defensa de edificaciones frente a incendios forestales en zonas de interfaz urbano forestal.

\section{PALABRAS CLAVE}

Riesgo de incendios forestales, Interfaz urbano-forestal, Infraestructura crítica, Estándares de construcción, Chile

\section{TECHNICAL PRESCRIPTIONS FOR HOUSING AND CRITICAL INFRASTRUCTURE IN THE WILDLAND-} URBAN INTERFACE TO FIRES: THE CASE OF SAN JOSÉ DE MAIPO, CHILE

\section{ABSTRACT}

In San José de Maipo, Metropolitan Region, Chile, the vulnerability of buildings was analyzed considering the impact of forest fires in interface areas. For this purpose, we collect population data, fire statistics, construction regulations, current legislation, interviews, workshops with communities, and, in general, extensive information from 2000 onward. With this background, we evaluate the areas with the highest number of residential buildings in forests and the main current construction regulations, as well as the characteristics of fire protection for different categories of construction: residential, schools, hospitals, and all types of critical infrastructure related to mitigation and protection features in case of disasters. Results show a high exposure of almost all categories of buildings. This occurs because constructions are located in areas of exposure with high vulnerability. Regarding to the existing buildings, proposals are offered to improve unsafe conditions in the most vulnerable areas. These proposals have resulted from a pilot experience in Chile, based on the results of the FONDEF Project it16i10003 (Chile), on protection and defense of buildings to forest fires in areas of wildland urban interface.

\section{KEYWORDS}

Wildfire risk, Wildland urban interface, Critical infrastructure, Building standards, Chile
1. Laboratorio de Incendios Forestales, Universidad de Chile, Santiago, Chile.

2. Facultad de Ciencias Forestales y de la

Conservación de la Naturaleza, Universidad de Chile, Santiago, Chile.

3. Instituto de la Vivienda INVI, Universidad de Chile, Santiago, Chile.

4. Programa de Reducción de Riesgos y Desastres CITRID, Universidad de Chile, Santiago, Chile.

*Autor de correspondencia: migcasti@uchile.cl

\section{RECIBIDO}

15 de abril de 2020

\section{ACEPTADO}

27 de mayo de 2020

\section{PUBLICADO}

1 de julio de 2020

\section{Formato cita}

Recomendada (APA): Castillo, M., Garay, R. \& Tapia, R. (2020).

Prescripciones Técnicas para Viviendas e Infraestructuras Críticas en Interfaz UrbanoForestal frente a Incendios: El Caso de San José de Maipo, Chile. Revista de Estudios Latinoamericanos sobre Reducción del Riesgo de Desastres REDER, 4(2), 71-84.

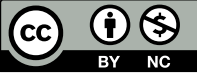

Todos los artículos publicados en REDER siguen una política de Acceso Abierto y se respaldan en una Licencia CreativeCommons Atribución-NoComercial 4.0 Internacional.

Revista de Estudios Latinoamericanos sobre Reducción del Riesgo de Desastres (REDER)

Diseño: Lupe Bezzina Tipografía: Hospital 


\section{INTRODUCCIÓN}

En los tiempos presentes, la ocurrencia de incendios forestales con características de desastres cobra cada día mayor relevancia mediática, especialmente por el aumento en la recurrencia de eventos de fuego bajo condiciones de severidad climática (Bowman et al., 2019; Castillo et al., 2019), por la disminución gradual de la humedad de los combustibles forestales, y también por los innegables efectos del cambio climático, sumado a ello, por la multiplicidad de actividades que se generan en territorios de alta demanda de bienes y servicios. La creciente actividad inmobiliaria, el deseo de disponer de una segunda vivienda alejada de las grandes ciudades, la permanente demanda de agua y la sobreexplotación de variados recursos naturales asociados al bosque, otorgan una sobredemanda de uso del suelo que expone a territorios a un mayor nivel de exposición y vulnerabilidad frente a la ocurrencia de incendios forestales, entre otro tipo de eventos (Castillo, 2006).

La creciente preocupación por mejorar los mecanismos de respuesta oportuna y atención de emergencias está ocasionando un aumento sostenido de la planta física para el combate de incendios forestales, prevaleciendo este criterio sobre la prevención. No obstante, aun cuando los países de ecosistemas mediterráneos como Chile dan mayor énfasis al planeamiento para el combate, no es menor el esfuerzo gubernamental que se está haciendo en materia local para fortalecer los programas de prevención (Castillo et al., 2012). Sin embargo, son escasas las experiencias que dan cuenta de medidas precautorias para preparar viviendas y otras infraestructuras en áreas vulnerables a la propagación del fuego.

Lo anterior se constituye en los tiempos presentes en una debilidad normativa para las actividades de construcción, y una brecha importante de investigación por cubrir. Estas debilidades se concentran en la precaria actualización de las normas y reglamentaciones constructivas, en el emplazamiento de las edificaciones, en los permisos para la concesión de espacios habitables y en general de falencias en los instrumentos locales de ordenación territorial que no colocan con especial fuerza y obligatoriedad la importancia de preparar los entornos habitables frente a la posibilidad de enfrentar emergencias derivadas de la propagación descontrolada del fuego.

Por todo lo anterior, este breve artículo refunde las principales experiencias en investigación desarrolladas en el vínculo del estudio de riesgos frente a desastres y las normas constructivas que se aplican para edificaciones localizadas en áreas de interfaz urbano forestal. Se coloca especial énfasis en la ocurrencia de incendios forestales, considerando a la comuna de San José de Maipo, en la Región Metropolitana, como estudio de caso. La investigación se basa en la experiencia del Proyecto FONDEF it16i10003, denominado Propuesta de Estándares en infraestructura crítica para edificaciones y viviendas localizadas en zonas de interfaz urbano-forestal, con el objetivo de minimizar el nivel de peligro en la propagación del fuego. Área piloto: comuna de San José de Maipo. Los resultados que aquí se exponen corresponde a dos tipos de enfoques: 'casa hacia adentro', fundamentalmente en el análisis de las normativas y tipologías constructivas con el propósito de mejorar las condiciones de defensa, y también el enfoque 'casa hacia afuera', principalmente relacionado a las condiciones externas o de emplazamiento en donde se encuentra localizada la infraestructura.

\section{Contexto de la vulnerabilidad en áreas de alto riesgo de incendios}

Existe un creciente interés en Chile por realizar estudios en zonas de contacto entre edificaciones y bosques, en donde se presenta una permanente actividad constructiva y con ello, actividad en el agente casuístico de ocurrencia y propagación de incendio forestales. Este interés es aún mayor si se considera la creciente necesidad por aplicar estrategias de mitigación local de los efectos atribuibles al cambio climático, y también a la permanente demanda de recursos naturales que de una u otra manera desencadena el aumento de la probabilidad de eventos catastróficos, entre ellos los incendios forestales (Castillo et al., 2014). Sin embargo, poco se conoce sobre las normas técnicas y resguardos necesarios a tomar en cuanto a diseño, autoprotección y gestión de zonas de peligro (Castillo, 2013). La normativa existente en materia de estándares y localización de construcciones habitables es débil, inconsistente y parcialmente regulada, lo que, sumado a escasez de temáticas regulatorias en cuanto a materialidad y criterios de armonía con el entorno, instalan la necesidad de estudiar sobre este tema, y proponer vías de desarrollo para el fortalecimiento de los sistemas de protección y atención de emergencias (Castillo et al., 2013). 


\section{MATERIALES Y MÉTODOS \\ Análisis territorial}

Para comenzar, lo que se propuso en este proyecto fue efectuar una evaluación territorial del problema de los incendios forestales en la comuna de San José de Maipo, para un área total aproximada cercana a 500 mil hectáreas. Para ello se evaluaron 22 localidades insertas en distintas condiciones de vulnerabilidad frente a incendios forestales (ver Figura 1). Se elaboró un primer análisis espacial, denominado 'Determinación de Prioridades de Protección', con el fin de identificar, evaluar y localizar las principales áreas con alta demanda en protección, y apoyar con ello la caracterización de las zonas críticas que pudieran contener áreas edificadas e infraestructura complementaria. Este paso inicial permitió posteriormente determinar la condición de exposición de diversos tipos de infraestructuras, las cuales fueron evaluadas desde el punto de vista normativo y técnico en cuanto a las normas constructivas actualmente vigentes en distintos cuerpos legales, entre ellos la Ordenanza General de Urbanismo y Construcción (OGUC) y prescripciones técnicas, muchas de ellas de carácter local no necesariamente normativas.

La evaluación territorial previa, basada en la calificación de tres categorías de protección contra incendios forestales, se ilustra mediante el desarrollo de un análisis multicriterio (ver Figura 2). En este territorio, poco más de 45 mil hectáreas presentan los valores más altos de exposición al fuego, y que comprometen casi la totalidad del área inmediata a las principales localidades de la comuna.

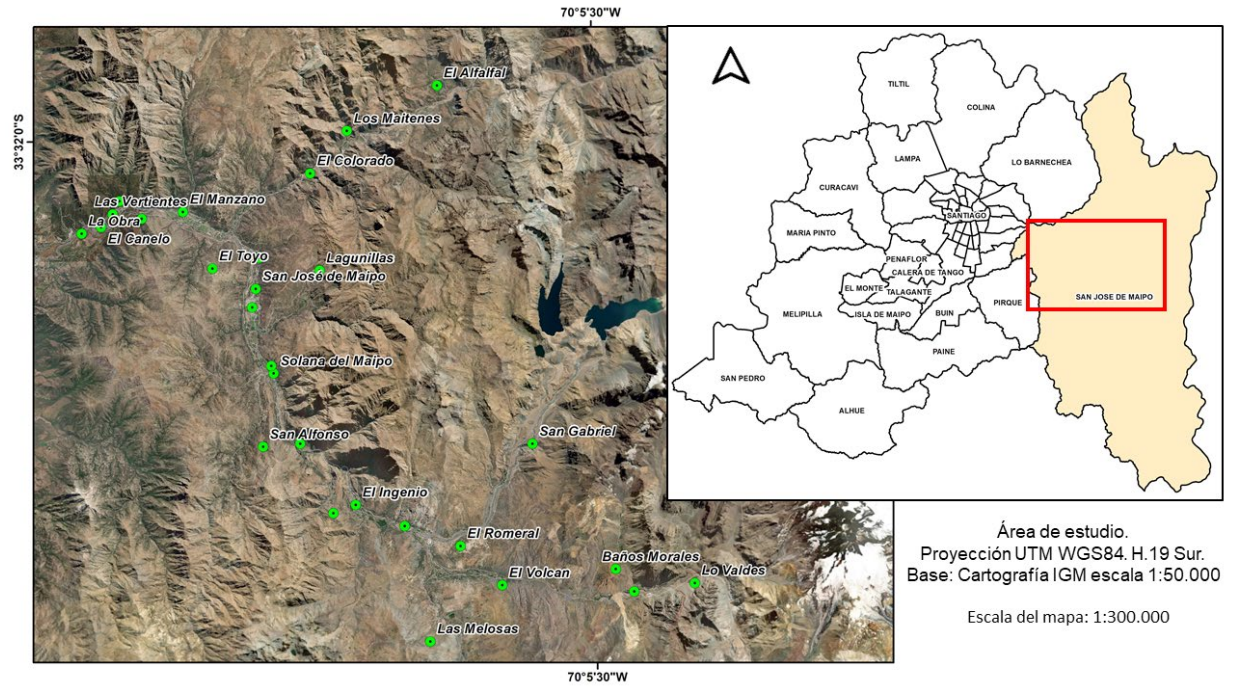

Figura 1. Área de estudio. comuna de San José de Maipo. Reg. Metropolitana de Chile Fuente: Autores, 2020

En el territorio antes indicado, existe una franja muy concentrada en las vías de acceso, especialmente en la principal ruta que permite la entrada y salida de vehículos y personas desde y hacia las distintas localidades de la comuna. Ello confiere una especial condición de riesgo asociado a la mayor probabilidad de ocurrencia de incendios forestales. Sumado a ello está presente la creciente actividad constructiva en zonas aledañas a bosques y sectores topográficos con alta peligrosidad para la propagación del fuego. Estos antecedentes y otros relacionados al comportamiento del fuego, clima, accesibilidad y características de la vegetación, entre otras, fueron incorporados a un análisis multicriterio para determinar las áreas con mayor exposición al fuego y en consecuencia, la evaluación de las áreas prioritarias sobre las cuales es necesario enfocar el análisis de la infraestructura crítica, sus emplazamientos y las normas por las cuales las edificaciones existen en áreas con distintos grados de exposición al fuego.

Este análisis multicriterio se compone de tres variables generales: Riesgo (probabilidad de iniciación de incendios); Peligro (conflictividad de los eventuales incendios que se originen); y Daño Potencial (pérdidas e impactos que podrían generarse con la propagación del fuego) (Castillo et al., 2015a, 2015b). A su vez, para cada variable general es necesario identificar las variables y subvariables específicas que la componen, las que deben definirse por su relevancia o influencia en el riesgo, peligro o daño potencial presente. Junto a la identificación de las variables, también es necesario definir su peso o ponderación (puntaje normalizado en una escala desde 0 a 100), de manera de permitir la valoración cuantitativa de cada una de las unidades de superficie que se 
consideren en la aplicación del método. Esta asignación de puntajes normalizados se inicia con las variables generales, con la precaución de que la suma de los tres valores de límites superiores de sus rangos sea igual a 100. Posteriormente, el valor fijado para cada una de las variables generales debe distribuirse entre todas las variables específicas seleccionadas para componerla. Para el caso de la comuna de San José de Maipo, se presenta a continuación la Figura 2, que contiene un esquema tentativo de variables y puntajes normalizados, el que ha sido elaborado sobre la base del conocimiento en terreno del lugar, de las entrevistas con personas y autoridades que habitan este territorio y también por integrantes y representantes de organizaciones y juntas de vecinos de la comuna, por ser ellos los primeros referentes en cuanto al conocimiento práctico del problema de los incendios forestales para este territorio. En particular, el análisis se ha centrado en las zonas pobladas y áreas aledañas a las localidades de El Canelo, Las Vertientes, La Obra, El Manzano, Guayacán, San José de Maipo, Lagunillas, El Melocotón, San Alfonso, El Ingenio, San Gabriel, El Volcán y Baños Morales, entre otras localidades dispersas en un territorio aproximado de 521 mil hectáreas.

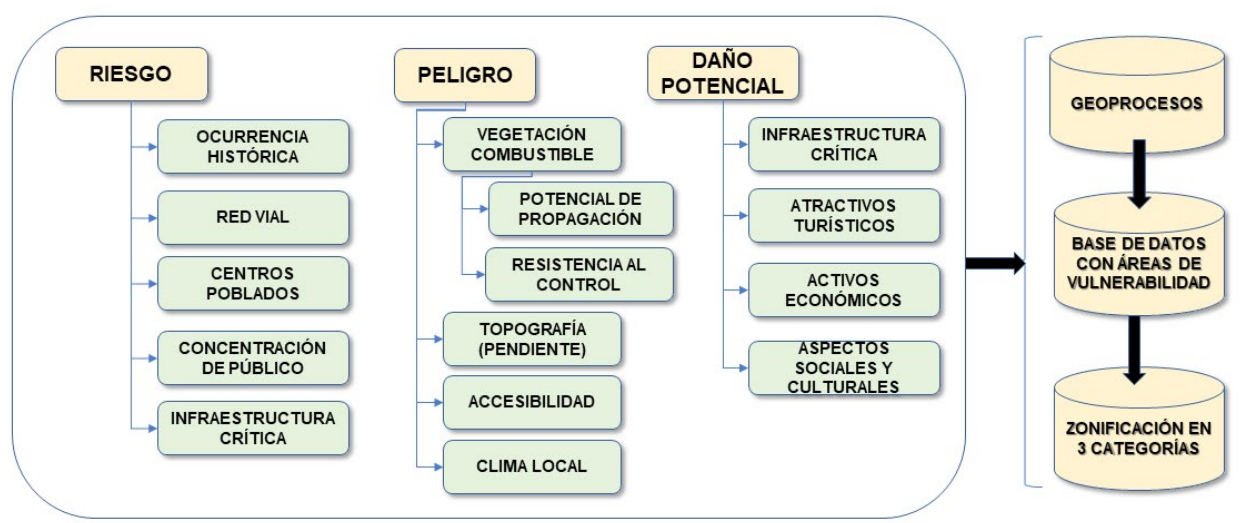

Figura 2. Esquema desarrollado para el proceso de determinación de zonas de vulnerabilidad, basado en análisis multicriterio. Comuna de San José de Maipo, Chile.

Fuente: Autores, 2020

En el caso del Riesgo, se asignaron 26 puntos (de un total de 100 posibles), los cuales fueron desagregados en 4 puntos para la ocurrencia histórica de incendios, 6 para la red vial, 6 para centros poblados, 5 para los sectores con concentración de público y otros 5 para la infraestructura crítica. El componente de Peligro fue valorado en 30 puntos, de los cuales 8 fueron asignados al potencial de propagación de la vegetación y 8 puntos más a la resistencia al control; también 6 puntos para la pendiente del terreno, 4 puntos para los sectores con difícil accesibilidad y 4 puntos para el clima local. Finalmente en lo concerniente al Daño Potencial, 44 puntos se distribuyeron en 10 para el valor económico de la infraestructura crítica, 12 para la diversidad de atractivos turísticos que podrían verse afectados por la propagación del fuego, y 11 puntos para los activos económicos y también para los aspectos culturales y sociales presentes en el área de estudio.

El análisis de peligro de incendios forestales toma especial relevancia por el aumento de la severidad de este tipo de eventos y la cada vez más estrecha relación de la propagación del fuego con la presencia o cercanía de centros poblados (Castillo, 2015; Costafreda et al., 2017). De esta manera, la importancia de estudiar la vulnerabilidad en zonas de interfaz urbano-forestal debe iniciarse, primero, con un catastro detallado de la condición de Peligro frente al comportamiento del fuego (CONAF, 2016). Para el desarrollo de la variable de Riesgo, mediante análisis satelital, se catastraron 3.889 edificaciones que se encuentran emplazadas en cerros con vegetación densa, y que por condiciones de conectividad presentan mayores dificultades de accesibilidad para los medios de extinción en caso de ocurrir un incendio forestal. Los resultados de este primer análisis permitieron, además de generar una expresión cartográfica de la vulnerabilidad, fue posible generar un Índice de Prioridad de Protección (IPP) que posteriormente se utilizó para focalizar las áreas más sensibles desde el punto de vista estructural y de localización de las edificaciones bajo riesgo de incendios.

\section{Análisis normativo}

Se recopilaron y analizaron las principales normas contenidas en Chile (ver Tabla 1), más las referencias establecidas por la Corporación Nacional Forestal para las recomendaciones técnicas en comunidades aledañas a bosques. Adicionalmente se recopilaron estándares extranjeros para 
establecer un nuevo enfoque normativo para el planeamiento de estructuras expuestas al impacto del calor radiante, en este caso, los efectos producidos por la cercanía de un incendio forestal. Para ello, en el desarrollo de las acciones 'casa hacia adentro', y 'casa hacia afuera', se enuncian los principales estándares y protocolos a recomendar:

\begin{tabular}{|c|c|c|c|}
\hline $\begin{array}{l}\text { Normativa y } \\
\text { Vigencia }\end{array}$ & $\begin{array}{l}\text { Organismos } \\
\text { Encargados }\end{array}$ & Descripción General de la Norma & $\begin{array}{l}\text { Tratamiento de Riesgos, Reconstrucción o } \\
\text { Planificación Urbana }\end{array}$ \\
\hline $\begin{array}{l}\text { Ley General de } \\
\text { Urbanismo y } \\
\text { Construcciones } \\
\text { LGUC (DS. W } 458 \\
\text { (V Y U.) 1976 a la } \\
\text { actualidad }\end{array}$ & $\begin{array}{l}\text { Ministerio de } \\
\text { Vivienda y } \\
\text { Urbanismo }\end{array}$ & $\begin{array}{l}\text { Contiene los principios, atribuciones, } \\
\text { potestades, facultades, responsabilidades, } \\
\text { derechos, sanciones y demás normas que rigen } \\
\text { a los organismos, funcionarios profesionales y } \\
\text { particulares en las acciones de planificación } \\
\text { urbana, urbanización y las construcciones, que } \\
\text { se desarrollen en todo el territorio de la nación } \\
\text { (Artículo } 1^{\circ} \text { y } 2^{\circ} \text { LGUC). (Ministerio de Vivienda y } \\
\text { Urbanismo, 2015) }\end{array}$ & $\begin{array}{l}\text { En el Artículo 2.1.17, se hace referencia a las 'reas } \\
\text { con riesgo de desastres. Conforme al artículo } 27 \\
\text { Título II "De la Planificación Urbana". En su } 2^{\circ} \\
\text { Capítulo, subdivide a la planificación urbana en } 4 \\
\text { niveles; nacional, regional, intercomunal y comunal. }\end{array}$ \\
\hline $\begin{array}{l}\text { Ordenanza } \\
\text { General de } \\
\text { Urbanismo y } \\
\text { Construcciones } \\
\text { (DS. No. } 47 \text { (V. } \\
\text { YU.)1992 a la } \\
\text { actualidad }\end{array}$ & $\begin{array}{l}\text { Ministerio de } \\
\text { Vivienda y } \\
\text { Urbanismo }\end{array}$ & $\begin{array}{l}\text { Reglamento de la LGUC, que contiene las } \\
\text { disposiciones reglamentarias de la ley, regula } \\
\text { procedimientos administrativos, el proceso de } \\
\text { la planificación urbana, la urbanización de los } \\
\text { terrenos, la construcción y los estándares } \\
\text { técnicos de diseño y construcción exigibles en la } \\
\text { urbanización y la construcción (artículo } 2^{\circ} \\
\text { LGUC) }\end{array}$ & $\begin{array}{l}\text { En Titulo } 2 \text { "De la planificación", profundiza su } \\
\text { referencia a los IPT e incorpora condiciones } \\
\text { específicas para los casos de las zonas de } \\
\text { remodelación y construcción obligatoria. Asimismo, } \\
\text { fija normas para urbanización y gestión y da las } \\
\text { pautas generales para las condiciones técnico- } \\
\text { urbanísticas. }\end{array}$ \\
\hline $\begin{array}{l}\text { Ley Orgánica } \\
\text { Constitucional de } \\
\text { Municipalidades } \\
\text { Ley No. } 18.695 \\
1988,2006 \text { a la } \\
\text { Actualidad }\end{array}$ & $\begin{array}{l}\text { Ministerio del } \\
\text { Interior, } \\
\text { Subsecretaria } \\
\text { Regional de } \\
\text { Desarrollo y } \\
\text { Administrativo }\end{array}$ & $\begin{array}{l}\text { Establece las disposiciones y atribuciones de las } \\
\text { municipalidades sobre el territorio, destinadas a } \\
\text { satisfacer las necesidades de la comunidad local } \\
\text { y asegurar su participación en el progreso } \\
\text { económico, social y cultural de la comuna. }\end{array}$ & $\begin{array}{l}\text { Grados de susceptibilidad al riesgo. Las limitaciones } \\
\text { que establezca el plan regulador o seccional se } \\
\text { definirán fundadamente en relación con el tipo y } \\
\text { grado de susceptibilidad del riesgo analizado en el } \\
\text { estudio de riesgos. Los grados de susceptibilidad } \\
\text { podrán ser definidos en: muy bajo, bajo, moderado, } \\
\text { alto o muy alto. }\end{array}$ \\
\hline $\begin{array}{l}\text { Artículo } 2.1 .17 \\
\text { OGUC, DS } 752001 \\
\text { (Modificado en } \\
2015 \text { y Vigente a la } \\
\text { actualidad) }\end{array}$ & $\begin{array}{l}\text { Ministerio de } \\
\text { Vivienda y } \\
\text { Urbanismo }\end{array}$ & $\begin{array}{l}\text { Definiendo en el Art. 2.1.17, las Áreas de Riesgo } \\
\text { cuando proceda un previo estudio específico, } \\
\text { por constituir un peligro potencial para los } \\
\text { asentamientos humanos. En dichas áreas se } \\
\text { determinarán zonas no edificables o de } \\
\text { condiciones restringidas de edificación. }\end{array}$ & $\begin{array}{l}\text { Grados de susceptibilidad al riesgo. Las limitaciones } \\
\text { que establezca el plan regulador o seccional se } \\
\text { definirán fundadamente en relación con el tipo y } \\
\text { grado de susceptibilidad del riesgo analizado en el } \\
\text { estudio de riesgos. Los grados de susceptibilidad } \\
\text { podrán ser definidos en: muy bajo, bajo, moderado, } \\
\text { alto o muy alto. }\end{array}$ \\
\hline $\begin{array}{l}\text { Ley } 19.300 \text { sobre } \\
\text { Bases Generales } \\
\text { del Medio } \\
\text { Ambiente } 2001 .\end{array}$ & $\begin{array}{l}\text { Ministerio del } \\
\text { Medio } \\
\text { Ambiente. }\end{array}$ & $\begin{array}{l}\text { Instaura el marco en el que se desarrollan las } \\
\text { tareas de seguimiento y fiscalización ambiental, } \\
\text { el cumplimiento de las normas y condiciones } \\
\text { sobre la base de las cuales se aprobaron los } \\
\text { Estudios y Declaraciones de Impacto Ambiental. }\end{array}$ & $\begin{array}{l}\text { No hace referencia específica a riesgos ni a } \\
\text { reconstrucción o planificación urbana. NO obstante, } \\
\text { para el caso de los IPT, en el DS N.O 95, se especifica } \\
\text { que los PRC contenían normas sobre. uso de suelo o } \\
\text { zonificación, localización del equipamiento. }\end{array}$ \\
\hline $\begin{array}{l}\text { Ley } 20.017 \\
\text { modifica Código } \\
\text { de Aguas } 2005\end{array}$ & $\begin{array}{l}\text { Ministerio de } \\
\text { Obras Públicas }\end{array}$ & $\begin{array}{l}\text { O.F.L. } 1.122 \text { de } 1981 \text { fija texto del Código de } \\
\text { Aguas. Regula el uso de cauces. Faculta al } \\
\text { Ministerio de Obras públicas (MOP) sobre la } \\
\text { vigilancia de obras en cauces. }\end{array}$ & $\begin{array}{l}\text { D.F.L. } 1.122 \text { de } 1981 \text { fija texto del Código de Aguas. } \\
\text { Regula el uso de cauces. Faculta al Ministerio de } \\
\text { Obras públicas (MOP) sobre la vigilancia de obras en } \\
\text { cauces. }\end{array}$ \\
\hline $\begin{array}{l}\text { Ley20.417que } \\
\text { modifica ley } 19300 \\
\text { sobre Bases } \\
\text { Generales del } \\
\text { Medio Ambiente } \\
2010 \text { a actualidad } \\
\end{array}$ & $\begin{array}{l}\text { Ministerio del } \\
\text { Medio } \\
\text { Ambiente }\end{array}$ & $\begin{array}{l}\text { Crea ministerio, servicio de evaluación } \\
\text { ambiental y superintendencia de medio } \\
\text { ambiente. }\end{array}$ & $\begin{array}{l}\text { Incorpora en la ley, la necesidad de que los IPT sean } \\
\text { sometidos a una Evaluación Ambiental Estratégica y } \\
\text { permite a la ciudadanía el acceso a la información } \\
\text { ambiental }\end{array}$ \\
\hline $\begin{array}{l}\text { Norma NCh-ISO } \\
\text { 31000:2012 }\end{array}$ & $\begin{array}{l}\text { Ministerio del } \\
\text { Interior }\end{array}$ & $\begin{array}{l}\text { Principios y directrices genéricas sobre la } \\
\text { gestión de cualquier tipo de riesgo. Pese a su } \\
\text { orientación genérica, no tiene como objeto } \\
\text { promover la uniformidad en la gestión del } \\
\text { riesgo. }\end{array}$ & $\begin{array}{l}\text { El diseño e implementación de planes y marcos de } \\
\text { trabajo deben considerar las diversas necesidades } \\
\text { de una organización, sus objetivos, su contexto o sus } \\
\text { activos y prácticas específicas utilizadas }\end{array}$ \\
\hline $\begin{array}{l}\text { Decreto } 1512 \\
\text { Aprueba Política } \\
\text { Nacional para la } \\
\text { Gestión del Riesgo } \\
\text { de Desastres } \\
\text { (GRD) } 2016 \text { a la } \\
\text { actualidad }\end{array}$ & $\begin{array}{l}\text { Ministerio del } \\
\text { Interior. } \\
\text { Ministerio de } \\
\text { Vivienda y } \\
\text { Urbanismo }\end{array}$ & $\begin{array}{l}\text { Fortalecimiento Institucional, Fortalecimiento } \\
\text { de los Sistemas de Monitoreo y Alerta } \\
\text { Temprana, Fomento de la Cultura de la } \\
\text { Prevención y el Auto aseguramiento, Reducción } \\
\text { de los Factores Subyacentes del Riesgo y el } \\
\text { Fortalecimiento de la Preparación ante los } \\
\text { Desastres para lograr una Respuesta Eficaz }\end{array}$ & $\begin{array}{l}\text { Pretende con esto, la coordinación territorial y } \\
\text { sectorial Fortalece la acción municipal por medio de } \\
\text { modificaciones a la Ley Orgánica de Municipalidades. } \\
\text { Asimismo, modifica y mejora el diseño de las } \\
\text { atribuciones en la emergencia, estableciendo } \\
\text { obligaciones de colaboración con instituciones } \\
\text { públicas y privadas. }\end{array}$ \\
\hline
\end{tabular}

Tabla 1. Instrumentos normativos asociados a la planificación urbana Fuente: Autores, 2020

Con este set de normas, el método contempló una revisión exhaustiva de las principales referencias internacionales que apuntan directamente a mejorar la protección en viviendas y enseres frente al fuego. Para ello se consideraron los estándares de Estados Unidos (Norma NFPA), normas europeas (EN e ISO), normas inglesas y euro-clases con énfasis en España. Todas estas normas se eligieron por su potencial adaptabilidad a las condiciones estructurales y de diseño actualmente presentes en la mayoría de los conglomerados habitacionales. Por cierto, el trabajo realizado requirió constantes revisiones sobre el actual grado de aplicabilidad de las normas chilenas sobre las actuales edificaciones emplazadas en San José de Maipo, junto con extraer las principales características de las restricciones extranjeras, siempre colocando foco en el tiempo de respuesta y resistencia al fuego. 


\section{RESULTADOS}

\section{Áreas vulnerables}

Las áreas de Primera Prioridad, es decir, aquellas zonas en donde se verifican los niveles más elevados de exposición frente al impacto de los incendios forestales corresponden a sectores asociados a asentamientos poblados y vías de comunicaciones (ver Figura 3). Los resultados derivados del análisis SIG, se complementan ahora con el análisis de zonas vulnerables estudiadas anteriormente por la Unidad de Manejo del Fuego de CONAF en 2016 para esta comuna, como una forma de analizar y validar los resultados de los análisis de Riesgo, Peligro y Daño Potencial desarrollados en el presente proyecto FONDEF. Las localizaciones están asociadas a la existencia o cercanía de infraestructuras y mayor carga de combustible vegetal susceptible de ser intervenida mediante acciones de silvicultura preventiva.

Es necesario indicar que la interfaz (zona de transición entre edificaciones y la vegetación), se encuentra altamente dispersa en la comuna, siendo necesario sectorizar -con previo análisis de la cartografía- el valor cuantitativo de riesgo, peligro y daño potencial para cada sector poblado. En tal sentido, se elaboró un catastro de todas las infraestructuras (principalmente casas) que se encuentran alejadas de los poblados principales, como un indicador de diagnóstico de la ocupación del territorio hacia zonas alejadas. Este fenómeno repercute en el aumento del nivel de peligro (proyecciones en la propagación del fuego), por consiguiente, hace más complejo el análisis por tratarse de un aumento en la dispersión de zonas altamente potenciales para la propagación del fuego.

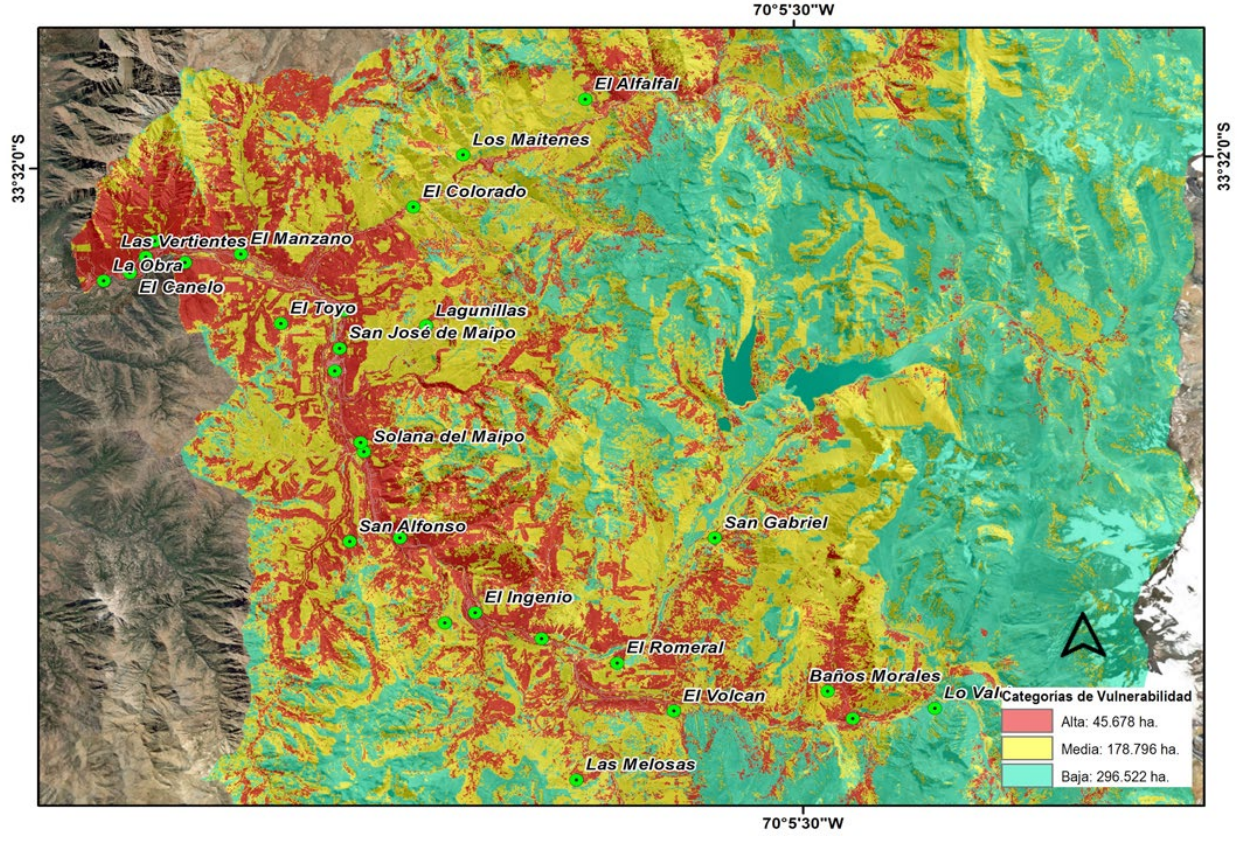

Figura 3. Representación espacial de las áreas con distintos grados de vulnerabilidad frente a incendios forestales. Las áreas más sensibles (en rojo) cubren prácticamente el 100\% de las áreas con presencia de edificaciones. Catastro a junio de 2019. Fuente: Autores, 2020

Las áreas anteriormente indicadas, están asociadas a la existencia de diversos tipos de carga vegetal combustible (principalmente matorral denso, pastizal y arbolado nativo denso y semidenso) y que, dentro del contexto de ignición e inflamabilidad, comprometen en su propagación los diversos tipos de infraestructura que puedan estar localizadas en cada una de las localidades presentes en esta comuna. Una extracción de los valores de Prioridad de Protección, siendo el valor máximo obtenido en el primer análisis de 72 sobre 100 (ver Tabla 2).

Más hacia la cercanía a zonas mayormente habitadas, existe una densidad relativamente homogénea en el trayecto de localización de estas estructuras, disminuyendo gradualmente desde el sector de El Ingenio hacia la cordillera. En las partes más bajas y de lomajes suaves, cercanos a intersecciones de caminos, la densidad de edificaciones es mayor, lo que concuerda con la distribución de la variable de Riesgo (probabilidad de ocurrencia de incendios) y con los mayores niveles de peligro (potencial de propagación y resistencia al control de los mismos). Estos resultados permitieron cerrar la primera evaluación respecto al problema espacial del peligro de incendios forestales, siendo la referencia para la tipificación de las estructuras que se encuentran insertas en estas áreas y también la caracterización desde el punto de vista preparatorio frente a la presencia de futuros incendios. 


\begin{tabular}{lccclccc}
\hline Localidad & Coord.X & Coord.Y & Valor & Localidad & Coord.X & Coord.Y & Valor \\
\hline La Obra & 362.168 & 6.282 .217 & 50 & Sn José Maipo Sur 2 & 374.896 & 6.274 .448 & 38 \\
Las Vertientes & 363.368 & 6.282 .865 & 37 & Lagunilla 1 & 376.224 & 6.275 .940 & 32 \\
El Canelo 1 & 365.350 & 6.283 .927 & 44 & Lagunilla 2 & 375.875 & 6.275 .050 & 40 \\
El Canelo 2 & 366.544 & 6.283 .959 & 40 & El Melocotón & 376.342 & 6.271 .157 & 60 \\
Las Lajas 1 & 368.725 & 6.283 .735 & 48 & San Alfonso & 378.002 & 6.267 .105 & 45 \\
Las Lajas 2 & 368.918 & 6.284 .128 & 41 & Vuelta del Padre 1 & 381.020 & 6.265 .557 & 38 \\
El Manzano 1 & 369.660 & 6.283 .646 & 39 & Vuelta del Padre 2 & 381.455 & 6.265 .039 & 45 \\
El Manzano 2 & 371.132 & 6.283 .057 & 43 & Vuelta del Padre 3 & 381.665 & 6.264 .769 & 49 \\
El Manzano 3 & 371.435 & 6.282 .785 & 30 & El Peumo 1 & 371.612 & 6.281 .574 & 37 \\
El Alfalfal 1 & 373.478 & 6.282 .039 & 27 & El Peumo 2 & 370.992 & 6.281 .843 & 38 \\
El Alfalfal 2 & 373.812 & 6.281 .381 & 55 & El Ingenio 1 & 381.771 & 6.262 .794 & 44 \\
Guayacán 1 & 374.599 & 6.280 .829 & 50 & El Ingenio 2 & 382.599 & 6.262 .516 & 39 \\
Guayacán 2 & 375.041 & 6.279 .953 & 48 & San Gabriel 1 & 384.136 & 6.261 .926 & 36 \\
San José de Maipo & 374.912 & 6.277 .440 & 48 & San Gabriel 2 & 385.600 & 6.261 .329 & 23 \\
Sn José Maipo Sur 1 & 375.346 & 6.275 .256 & 37 & San Gabriel 3 & 386.077 & 6.260 .935 & 46 \\
\hline
\end{tabular}

Tabla 2. Valor asociado a la Prioridad de Protección para cada una de las localizaciones determinadas prioritarias. Valor máximo de referencia: 72

Fuente: Autores, 2020

\section{Análisis normativo. Acciones 'casa hacia adentro'}

En casa hacia adentro se analizaron las normativas constructivas actualmente vigentes, respecto a las características presentes en las estructuras interiores de las edificaciones. Se evaluó con un índice de vulnerabilidad la exposición de las infraestructuras para todas las localidades desde el punto de vista de la vulnerabilidad (ver Tabla 2), incluyendo a centros hospitalarios o de salud, unidades policiales, bomberos, establecimientos educacionales, indicadores asociados al turismo y la ponderación final en un indicador multicriterio llamado Índice Integrado de Seguridad (IIS). Este índice fue desarrollado mediante una planilla Excel la cual se coloca a disposición de los gestores de información territorial, previa capacitación y entrenamiento del modo de uso. La información acá obtenida es válida para distintas localidades y períodos determinados por las autoridades de acuerdo a su planeamiento de prioridades en materia de prevención de desastres. Como extensión, nuestra metodología también es válida para ser replicada en otras comunas, siendo únicamente necesaria la adaptación de las variables iniciales para el diagnóstico de la condición de peligro. Como resultado de este paso, en modalidad 'casa hacia adentro', se entrega entonces: un índice integrado IIS, más un reporte de las principales medidas técnicas de tipo constructivas necesarias de considerar para mejorar el estado actual de las infraestructuras y los futuros emplazamientos dentro de la comuna. Esta información es compartida con las comunidades, para el conocimiento y toma de razón para la ejecución de acciones - si procede - en materia de mejoramiento de infraestructura. Las normativas extranjeras analizadas para el reforzamiento de los instrumentos locales actualmente vigentes fueron las normas NFPA de Estados Unidos, Norma Australiana y Norma Europea. En cada una de ellas de extrajeron las características atingentes a la materialidad exterior y los requisitos de protección frente a la inflamabilidad de mobiliario y enseres. Estas características técnicas fueron consideradas para complementar las existentes en las normativas chilenas (ver Tabla 3).

\begin{tabular}{l}
\hline NCh 382 of. 98. Sustancias peligrosas - Terminología y clasificación general \\
\hline NCh 1411 of. 78. Identificación de riesgos de materiales \\
\hline NCh 2120 /1 al 9/ of. 89. Sustancias peligrosas - Parte 1 al 9: Clases 1 al 9 \\
\hline NCH 2137 of. 92. Sustancias peligrosas - Embalajes/envases \\
\hline NCh 933. Terminología general \\
\hline NCh 934. Clasificación de fuegos \\
\hline NCh 935/1. Ensaye de resistencia al fuego - Parte 1: Elementos de construcción general \\
\hline NCh 935/2. Ensaye de resistencia al fuego - Parte 2: Puertas y otros elementos de cierre \\
\hline NCh 2209. Ensaye del comportamiento al fuego de elementos de construcción vidriados \\
\hline NCh 1914/1. Determinación de la no combustibilidad de materiales de construcción \\
\hline NCh 1993. Clasificación de los edificios según su carga combustible \\
\hline NCh 1974. Pinturas - Determinación del retardo al fuego \\
\hline NCh 1977. Comportamiento de revestimientos textiles a la acción de una llama \\
\hline NCh 1979. Comportamiento de telas a la acción de una llama \\
\hline NCh 2189. Seguridad - Señalización de edificios - Condiciones básicas \\
\hline
\end{tabular}

Tabla 3. Normas chilenas de seguridad en incendios (DS.594/99 MINSAL Chile) Fuente: Autores, 2020

Estas normativas contemplaron el análisis para distintos tipos de edificaciones: viviendas, colegios, hospitales y otros centros médicos de asistencia, y también edificaciones aisladas. El cruce de esta información con los aspectos normativos factibles de adaptar de las normas internacionales, dieron como resultado un protocolo de recomendaciones basado en la distancia existente entre el 
estado actual de las infraestructuras de la comuna, y las proyecciones de mejoramiento basadas en las directrices internacionales, con el fin de elevar el estándar preparatorio de edificaciones frente a incendios forestales, como también mejorar las condiciones actuales de defensa en las viviendas ya instaladas en áreas de interfaz urbano-forestal. Así lo propone también CONAF en su manual de preparación de viviendas frente a incendios (CONAF, 2015).

Con todos estos antecedentes, tanto los nacionales como los extranjeros, fueron considerados para elaborar un protocolo de adaptabilidad informada respecto a los aspectos normativos necesarios de incorporar a los estándares actuales de protección contra el fuego. Para este trabajo, se consideró la referencia espacial dada por la calificación de las zonas de vulnerabilidad, y con ello, las propuestas de aseguramiento preventivo frente al daño. En este sentido, para el caso de incendios forestales de interfaz, tal como se define en el estándar Community Wildfire Safety Through Regulation de National Fire Protection Association, las acciones de protección se ubican a distintos niveles, a saber: escala comunitaria, escala vecinal, escala propiedad individual (casa hacia afuera), y la estructura misma (casa hacia adentro). Para responder a la pregunta, de cómo adoptar Regulaciones frente a incendios forestales, los distintos países optan por medidas de mitigación para abordar el riesgo que pueden generar fuertes discusiones y a menudo opiniones conflictivas dentro de una comunidad. Un acercamiento exitoso a las regulaciones debe anticipar y abordar este riesgo de adaptación y hacerse cargo de este proceso mediante una secuencia de acciones, las cuales fueron aplicadas para San José de Maipo (ver Figura 4).

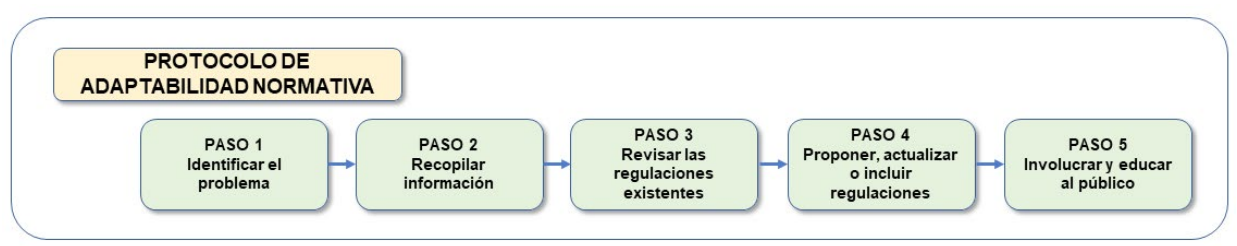

Figura 4. Protocolo de adaptabilidad normativa, aplicable a las edificaciones en San José de Maipo Fuente: Autores, 2020

Un tema relevante, pero no abordado en términos normativos se refiere a que las comunidades necesitan decidir a qué escala (s) están dispuestos y son capaces de regular. Por lo que esta metodología, intenta abordar distintos niveles de solución que pueden ser más cercanos a las personas, pues les permite comprender en primer lugar, la importancia de la seguridad de su vivienda, es decir lo que corresponde a la edificación unitaria y a las estructuras de los elementos.

\section{Análisis del comportamiento del fuego. Acciones 'casa hacia afuera'}

Consistió principalmente en proponer intervenciones conducentes a proteger las viviendas y estructuras aledañas frente al efecto del calor radiante producto de la acción de las llamas, en un perímetro variable. Estas intervenciones son variables en cuanto a tipo, intensidad, localización y oportunidad. Las referencias de estas actividades están dadas en primera instancia por el conocimiento a los gestores municipales y representantes locales sobre su valor puntual del valor de vulnerabilidad frente al fuego casa hacia afuera en la localidad o grupo local de viviendas, y posteriormente establecer las acciones conducentes a controlar el factor de peligro sobre la vegetación combustible.

En el desarrollo de esta pauta, fue necesario comunicar a los residentes de viviendas y su infraestructura asociada (bodegas, escombros, pastos, ramas secas, árboles cercanos), acciones preventivas las cuales deben ser ejecutadas y supervisadas por personal municipal. Una medida ineludible de realizar en sectores que interactúan construcciones con pastizales, matorrales o árboles, y considerando además la densidad de árboles, espaciamientos y el efecto de la pendiente, se establece mediante el cálculo del comportamiento del fuego.

Para este propósito se realizó la modelación basada en las ecuaciones del Sistema KITRAL, desarrollado por el Laboratorio de Incendios Forestales de la Universidad de Chile (Castillo, 1998), con lo cual se han generado tablas y coeficientes específicos que dan cuenta del valor de la velocidad de propagación del fuego en cada tipo de vegetación, y también el grado de dificultad o resistencia al control que expone cada tipo vegetacional frente a la realización de una línea de control.

Por lo anterior, en estos resultados se han considerado tres parámetros técnicos: la carga de vegetación fina y muy fina (factor ' $w$ '), incluyendo ramas y desechos muertos hasta 1,7 metros desde la rasante o piso vegetal (expresado en toneladas de biomasa por hectárea, normalizado al promedio de la unidad analizada), el poder calorífico (factor ' $\mathrm{H}$ ') o cantidad de kilocalorías 
capaz de emitir el cuerpo vegetal producto de la combustión, y finalmente el factor de velocidad de propagación lineal del fuego (factor ' $r$ ') que se desarrolla dependiendo del tipo de vegetación afectada; este último parámetro se expresa en metros de avance por segundo. La combinación de éstos tres factores se han normalizado bajo un escenario medio para el cálculo de estos parámetros (ver Tabla 4), dando como resultado la expresión de la intensidad calórica de las llamas que se desarrollan en forma dinámica conforme va variando en su recorrido por la pendiente y cambios en la velocidad y dirección del viento. Para efectos de cálculo se definió escenario medio como aquel período de máxima probabilidad de ocurrencia de incendios forestales para el área de estudio, y que va asociado a condiciones meteorológicas específicas para las variables de temperatura, humedad relativa del aire, velocidad y dirección del viento y la condición de sequía.

\begin{tabular}{ll}
\hline Parámetro & Umbral (escenario máximo) \\
\hline Temperatura del aire & $30^{\circ} \mathrm{C}$ \\
\hline Humedad relativa del aire & $30 \%$ \\
\hline Velocidad del viento & $30 \mathrm{~km} / \mathrm{h}$ \\
\hline Dirección del viento & $\mathrm{Sur}\left(180^{\circ}\right)$ \\
\hline Contenido de humedad de la vegetación fina & $7,5 \%$ \\
\hline Pendiente & $30 \%$ \\
\hline Nivel de sequía & 30 días previos sin precipitaciones \\
\hline
\end{tabular}

Tabla 4. Condiciones ambientales preestablecidas para el proceso de cálculo de la intensidad calórica de las llamas. El instructivo plantea estos valores como condición extrema de comportamiento del fuego, otorgando un margen de seguridad máximo para la implementación de las acciones preparatorias Fuente: Autores, 2020

Con los datos sobre factor pendiente y condiciones de la vegetación de acuerdo al modelo asociado, es posible entonces asociar el factor I de intensidad, al ancho de barreras necesario de aplicar para diferentes modelos de combustibles y también para distintas condiciones de inclinación del terreno, contextualizado a las zonas de interfaz urbano-forestal, con lo cual sus resultados también pueden ser aplicados asociando el nivel de protección y de vulnerabilidad potencial que tenga cada área en relación al impacto potencial de las llamas. Se presentan a continuación los antecedentes usados y los resultados de los procesos ejecutados para definir la anchura mínima de cortafuegos y en diferentes modelos de combustibles y condiciones ambientales preestablecidas, considerando los efectos de la radiación horizontal y la convección (emisiones de pavesas de grosor medio) generados por eventuales frentes de propagación de incendios forestales.

\begin{tabular}{lccc}
\hline Modelo de combustible & $\begin{array}{c}\text { Carga [w] } \\
\left(\text { ton ha } \mathbf{~}^{-1}\right)\end{array}$ & $\begin{array}{c}\text { Poder calorífico [H] } \\
\left(\mathrm{kcal} \mathrm{m}^{-1} \mathbf{s}^{-1}\right)\end{array}$ & $\begin{array}{c}\text { Factor de } \\
\text { propagación [r] } \\
\left(\mathrm{m} \mathrm{s}^{-1}\right)\end{array}$ \\
\hline Matorrales y Arbustos Semidensos - Densos & 2,923 & 4693 & 0,007603 \\
\hline Pastizales, Estratos Herbáceos Densos & 0,918 & 3928 & 0,010235 \\
\hline Formaciones Vegetales muy densas & 3,529 & 5087 & 0,009234 \\
\hline Plantación de Pino Radiata Adulto con manejo & 3,714 & 4870 & 0,003255 \\
\hline Plantación de Eucaliptus sp Adulto & 2,742 & 4816 & 0,005429 \\
\hline
\end{tabular}

Tabla 5. Tratamientos recomendables de acuerdo a los factores que regular la emisión de calor radiante, en función de la pendiente Fuente: Autores, 2020

Con los factores descritos (ver Tabla 5), se determinaron cuatro factores del comportamiento del fuego (Julio et al., 1995a), utilizando el sistema KITRAL: el modelo de combustible (Fmc), contenido de humedad de los mismos (Fch), Factor velocidad del viento (Fv) y factor pendiente del terreno (Fp). La expresión numérica utilizada fue VPL (velocidad de propagación lineal del fuego) $=(F m c)^{\star}(F c h) *(F p+F v)$, y cuya salida entrega la velocidad de avance del fuego en metros por segundo o por minuto (Julio et al., 1995b) (ver Tabla 6), dependiendo de cómo estén expresadas las unidades.

\begin{tabular}{ccc}
\hline Pendiente $(\%)$ & VPL $\left(\mathrm{m} \mathrm{s}^{-1}\right)$ & $\mathrm{I}\left(\mathrm{Kcal} \mathrm{m}^{-1} \mathbf{s}^{-1}\right)$ \\
\hline 10 & 1,21272 & 2510 \\
\hline 20 & 1,24193 & 2571 \\
\hline 30 & 1,27439 & 2638 \\
\hline 50 & 1,34903 & 2793 \\
\hline 70 & 1,43450 & 2969 \\
\hline 90 & 1,53186 & 3171 \\
\hline
\end{tabular}

Tabla 6. Variación de la velocidad de avance del fuego e intensidad calórica del frente principal de avance de las llamas en función de la pendiente del terreno

Fuente: Autores, 2020

Como referencia, los valores de VPL e I pueden variar ostensiblemente dependiendo de las condiciones locales del entorno de propagación. Castillo (2013), en un estudio de la propagación del fuego en viviendas de interfaz urbano-forestal en Valparaíso, reporta velocidades de propagación superiores a 10,27 $\mathrm{ms}^{-1}$ cuando el fuego corre libremente por pastizales y velocidades del viento 
superiores a $45 \mathrm{kh}^{-1}$. En estas condiciones, el fuego desarrolla importantes incrementos en perímetro y superficie. En cuanto a la liberación de calor radiante, se han reportado instantes con $16.000 \mathrm{Kcal} \mathrm{m}^{-1} \mathrm{~s}^{-1}$, siendo condiciones extremas de corto tiempo de permanencia, dependiendo de la velocidad de combustión de los materiales combustibles, entre ellos las casas. Las viviendas de material ligero desarrollan normalmente los mayores montos de energía y altura de llamas. Con los valores de referencia sobre la velocidad de avance y el monto energía por metro y por segundo, es posible determinar valores de referencia para la construcción de barreras, ya sean cortafuegos o corta combustibles, siendo posible además establecer medidas intermedias que permitan aumentar el tiempo de reacción frente a la posibilidad de un salto de fuego desde un extremo de frente de propagación hacia una sección opuesta amenazada por el fuego.

La determinación del ancho del cortafuego a realizar en las áreas cercanas a viviendas, integra los efectos combinados de la radiación y la convección que generan las llamas, las que a su vez dependen del nivel de la intensidad calórica lineal de ese frente de avance. En dicho planteamiento, hemos incorporado estándares de comportamiento del fuego desarrollados por Castillo (2013) en áreas de interfaz localizadas en Valparaíso, basados en el cálculo de la intensidad calórica del fuego. Adaptando estos datos a los encontrados en San José de Maipo, se considera que, dependiendo del ancho del cortafuego, en su lado receptor de energía calórica se podría generar un encendido equivalente a una intensidad calórica lineal de alrededor de $100\left(\mathrm{kcal} \mathrm{m}^{-1} \mathrm{~s}^{-1}\right)$ en un lapso de 1 a 3 minutos $y$, en un período de 3 a 5 minutos en los casos que se pueda provocar un foco de una intensidad calórica lineal cercana a $50\left(\mathrm{kcal} \mathrm{m}^{-1} \mathrm{~s}^{-1}\right)$. Se exponen valores de referencia (ver Tabla 7), considerando los modelos de combustibles presentes en el área de estudio, y que de acuerdo a los datos de la Tabla 5, presentamos los resultados basados en la dominancia de tres formaciones predominantes: matorrales y arbustos semidensos a densos, pastizales y estratos herbáceos densos, y por último, otras formaciones vegetales muy densas de variada composición florística. De estos combustibles se evaluaron las proporciones de cada tipo vegetacional para luego extraer los valores de intensidad calórica, carga y potencial de propagación. Posteriormente en la interfaz analizada, se determinaron los niveles de intensidad calórica de acuerdo a las fórmulas de Albini, con los datos de pendiente, viento, temperatura y humedad indicados anteriormente.

\begin{tabular}{ccccccc}
\hline \multirow{2}{*}{$\begin{array}{c}\text { Ancho del } \\
\text { cortafuego }\end{array}$} & \multicolumn{5}{c}{ Intensidades calóricas lineales recibidas según niveles de pendiente } \\
\cline { 2 - 6 }$(\mathrm{m})$ & $\mathbf{6}$ & $\mathbf{2 0}$ & $\mathbf{3 0}$ & $\mathbf{5 0}$ & $\mathbf{7 0}$ & $\mathbf{9 0}$ \\
\cline { 2 - 6 } & 112,65 & 116,01 & 118,66 & 121,02 & 124,54 & 129,91 \\
\hline 5 & 71,11 & 74,75 & 76,03 & 79,42 & 84,68 & 100,03 \\
\hline 6 & 50,07 & 53,32 & 55,46 & 57,98 & 62,88 & 66,42 \\
\hline 7 & 38,55 & 41,68 & 43,36 & 46,91 & 48,77 & 50,15 \\
\hline 8 & 31,04 & 31,74 & 32,58 & 34,45 & 37,07 & 40,05 \\
\hline 9 & 25,22 & 25,93 & 27,42 & 28,07 & 30,03 & 32,52 \\
\hline 10 & & & & & & \\
\hline
\end{tabular}

Encendido en 1 a 3 minutos Encendido en 3 a 5 minutos

Tabla 7. Niveles de Intensidad Calórica Lineal Recibida, según la pendiente y el ancho del cortafuego Fuente: Autores, 2020

Los valores anteriores pueden ser ampliados (cálculos sucesivos para anchos superiores a 10 metros y con ello un mayor tiempo de retardo en la combustión inicial), hacia la vecindad de las viviendas, generándose mayor tiempo de encendido en la medida que la intensidad calórica lineal decrece en función de la distancia. En estos cálculos se consideraron las estructuras aéreas y biomasa aérea que eventualmente podrían cruzar la barrera de defensa, generando transporte de materiales incandescentes y con ello la continuidad del avance de las llamas. De acuerdo a las recomendaciones y estudios generados en mesas técnicas sobre continuidad de combustibles, y los resultados de la modelación del comportamiento del fuego utilizando como base los cálculos anteriormente indicados, se plantean anchos técnicos que variarán entre un umbral mínimo de 5 metros (datos de la Tabla 7), hasta valores de 24-25 metros para el caso de matorrales altos y densos con pendientes superiores a $50 \%$. Estos valores constituyen referencias que permiten programar las labores preventivas de tratamientos al combustible vegetal.

De acuerdo a los datos de intensidad calórica descritos en la Tabla 7 para la carga de combustible mixto cercana a viviendas, se exponen prescripciones para las principales situaciones encontradas: pastizales densos a semidensos: 9 a 10 metros para lograr un retardo de encendido superior a 5 minutos, arbustos y arbolado (de más de 1 metro de altura desde el suelo): 20 metros para lograr un retardo entre 5 a 10 minutos. Desde el cortafuego hacia la zona forestal, se deberá 
establecer una faja corta combustible de 80 metros de ancho mínimo, para asegurar tiempos mayores de respuesta para los medios de extinción, debido a la necesidad de desplazamiento hacia el interior de las áreas amagadas por el fuego. Esta faja deberá mantenerse con estrato herbáceo, matorrales ralos bajos o arbolado de baja densidad. En el caso de presencia de plantaciones forestales, el corta combustible deberá producir discontinuidad horizontal y vertical mediante raleos y podas, respectivamente.

\begin{tabular}{ll}
\hline Aspecto & Descripción \\
\hline Pendiente & Porcentaje medio de pendiente cercana a la vivienda \\
\hline Continuidad horizontal & Continuidad horizontal de la masa forestal \\
\hline Ancho de camino & Valor medio de ancho de caminos interiores \\
\hline Altura de árboles & Altura media de árboles a orilla de camino \\
\hline Presencia de intervenciones & Podas, raleos, desbroces, manejo de tocones \\
\hline Presencia y altura de pastos & Altura media de pastos finos y arbustos delgados \\
\hline Densidad de dosel inferior & Densidad de estratos intermedios e inferiores \\
\hline Contacto entre especímenes & Cruce de ramas entre especímenes \\
\hline Continuidad vertical & Continuidad vertical de la masa arbórea y/o arbustiva \\
\hline Presencia de escombros y/o basura & Combustible grueso cercano a vivienda \\
\hline Presencia de desechos orgánicos & Pastizales finos, desechos vegetales secos \\
\hline Leña u otros almacenamientos & Pilas de leñas y/o restos de madera adosadas a la vivienda \\
\hline Tendidos eléctricos externos & Red de cables que podrían intersectar ramas \\
\hline
\end{tabular}

Tabla 8. Aspectos de terreno a considerar para las intervenciones en la vegetación colindante a viviendas -basado en Castillo et al. (2016) y De Luis et al. (2004).

Fuente: Autores, 2020

En el caso de raleos, se deberá evitar el cruce de copas y en el caso de las podas, ésta afectará al $40 \%$ de la altura de los individuos, hasta un máximo de 8 metros. Además, se deberá reducir el material combustible bajo dosel, considerando el manejo del estrato arbustivo y herbáceo. Se deberán efectuar actividades de mantención periódica tanto en el cortafuego, como en la faja corta combustible, las que deberán permanecer libres de desechos domésticos, basura u otros materiales combustibles.

En cuanto a la presencia de caminos, éstos deben ser aprovechados como cortafuegos permanentes, y que por lo tanto al no tener prácticamente costos de mantención, pueden ser reforzados con prescripciones a la vegetación de borde. Para ello se propone una faja adicional de 10 metros a cada lado del camino con raspado hasta el suelo mineral, siguiendo la premisa de cálculo de la Tabla 7 (retardo en el tiempo de encendido), para luego continuar con una reducción de carga de árboles y arbustos mayores desde los 10 metros de la primera faja hacia el interior (ancho de 15 metros); en esta segunda faja se aplicarán podas, raleos y cortas de liberación para reducir la carga gradualmente hacia el eje del camino. (valores acumulativos, es decir, 25 metros de intervención), mientras que para vías secundarias se recomienda 5 metros de cortafuegos y otros 10 adicionales de corta combustibles. La faja corta combustible deberá considerar una poda que afecte el $40 \%$ de la altura de los individuos, hasta un máximo de 8 metros, raleo para evitar el cruce de copas y la reducción de combustibles. El cortafuegos a borde de caminos y la faja corta combustible, estarán sometidas a mantención periódica, debiendo permanecer libre de material combustible, residuos y asentamientos humanos. Estas prescripciones en muchos casos suponen un alto costo y organización de las cuadrillas de trabajo, con lo cual deberán priorizarse aquellas áreas o secciones que presenten mayor densidad de vegetación presente en este rango de distanciamiento (0-25 metros).

\section{DISCUSIÓN}

Los valores de intensidad y anchos de cortafuegos, como asimismo las prescripciones silvícolas pueden ser ampliados hacia la vecindad de las viviendas, generándose mayor tiempo de encendido en la medida que la intensidad calórica lineal decrece en función de la distancia. No olvidar que sobre estos cálculos se deben considerar las estructuras aéreas y biomasa aérea que eventualmente podrían cruzar la barrera de defensa, generando transporte de materiales incandescentes y con ello la continuidad del avance de las llamas.

En la zona de interfaz urbano-forestal se deberán establecer criterios de despeje de material vegetal, de acuerdo a las definiciones indicadas previamente. Desde el cortafuego hacia la zona forestal, se deberá establecer una faja corta combustible de ancho variable, la cual deberá cautelar generar el efecto cuña hacia el interior, misma modalidad aplicable a contención del fuego basada en la presencia de caminos. En el caso de existir mezclas de matorral, plantaciones y arbolado nativo, deberá evaluarse la continuidad horizontal y vertical, dependiendo de las condiciones 
técnicas y económicas factibles de implementar. En el caso de plantaciones implica considerar la modalidad de raleos, limpias y podas a aplicar en cada caso.

En el caso de raleos, se deberá evitar el cruce de copas y en el caso de las podas, si bien es cierto existen prescripciones técnicas de altura para el corte de ramas (normalmente hasta 5-6 metros) que aplican para empresas forestales con maquinaria y personal especializado, el umbral de despeje vertical para pequeños propietarios se recomienda dejarlo en 3 metros desde el suelo, abordando con ello la carga principal de material combustible en los estratos inferiores, incluyendo los desechos generados de la poda, y por donde generalmente avanza el fuego en sus estados iniciales antes de coronar. Adicionalmente se deberá reducir el material combustible bajo dosel, considerando el manejo del estrato arbustivo y herbáceo. Se deberán efectuar actividades de mantención periódica tanto en el cortafuego, como en la faja corta combustible, las que deberán permanecer libres de desechos domésticos, basura u otros materiales combustibles.

En cuanto a la presencia de caminos, de acuerdo a los estudios de caso desarrollados en diversidad de combinaciones entre altura de árboles, densidad de la vegetación y ancho de los caminos, se recomienda que para las vías asfaltadas de dos o más pistas, se efectúe como mínimo 10 metros de cortafuegos y otros 15 adicionales de corta combustible (valores acumulativos, es decir, 25 metros de intervención), mientras que para vías secundarias se recomienda 5 metros de cortafuegos y otros 10 adicionales de corta combustibles.

Respecto a la dimensión tecnológica y la dimensión normativa, éstas se unen para el establecimiento de protocolos preventivos de actuación frente a futuras emergencias derivadas de incendios forestales. Correspondió a un trabajo que contempló, por un lado, el desarrollo de un Índice Integrado de Seguridad (IIS) que da cuenta del estado específico de las distintas infraestructuras existentes en la Comuna, en particular, los establecimientos educacionales, de salud, cuarteles de policía, bomberos e infraestructura turística, todos los anteriores, analizados desde distintos tipos de vista normativos y de exposición frente al fuego. Para ello, el enfoque 'casa hacia adentro' y 'casa hacia afuera', permitió generar pautas operativas basadas en la revisión de estándares impuestos por normativas extranjeras, tales como NFPA de Estados Unidos, pero que previamente fueron detalladamente revisadas, interpretadas y adaptadas como propuestas de diseño a la realidad de edificaciones, tanto existente como proyectada en las áreas potencialmente planificada para futuras construcciones.

Por el lado de la dimensión social colectiva, se propusieron distintas pautas operativas para el fortalecimiento de la red de gestión preventiva y preparatoria a nivel de grupos de viviendas, sus organizaciones vecinales y el vínculo de ellas con las autoridades municipales. Este paquete de medidas fue discutido y consensuado en distintas asambleas participativas en donde mediante técnicas de lluvia de ideas, muestreo de opiniones y resúmenes consolidados, fue posible obtener las principales demandas de la comunidad frente al tratamiento preventivo, especialmente de la vegetación combustible, y de manera muy particular, una revisión de la infraestructura de apoyo que juega un rol preponderante para las acciones de intervención, traslado y evacuación. Esta discusión formó parte del protocolo, cuyos principales elementos se indican a continuación.

\section{CONCLUSIONES}

De acuerdo al análisis multicriterio que permite la identificación de las áreas con mayor nivel de peligro frente a incendios forestales, se determinó que las zonas más sensibles para el área en estudio, contemplan una superficie aproximada de 45 mil hectáreas, y que incluyen la totalidad de las localidades de esta comuna.

Basado en el catastro de viviendas consolidadas, más aquellas que se encuentran emplazadas en cerros (cercano a 4 mil), presentan una diversidad de situaciones en cuanto a materialidad y una evidente irregularidad en las normas constructivas, lo que acentúa fuertemente el problema de la vulnerabilidad.

Aun cuando no se trató en detalle en esta investigación, un aspecto clave a considerar en la priorización de áreas con máxima vulnerabilidad, son los tiempos de respuesta frente a emergencias de incendios. En tal sentido, el trabajo colectivo con las distintas comunidades y representantes de juntas de vecinos dio como resultado un ajuste de las áreas prioritarias a donde realmente se necesitan los recursos para establecer las medidas prescriptivas y cambios normativos para los enfoques casa hacia adentro y casa hacia afuera. 
La delimitación de tipologías constructivas basada en un detallado catastro, es un factor clave para dimensionar en su conjunto, el estado actual de las infraestructuras en San José de Maipo y sus proyecciones de mejora. En esta delimitación se consideraron las escuelas y liceos, establecimientos de salud, viviendas en cascos urbanos y aquellas alejadas hacia los cerros, como también infraestructura de turismo y de atención de servicios básicos para la comunidad: locaciones de bomberos, carabineros, servicios prioritarios de salud, Municipio y otras instalaciones que pudieron ser evaluadas desde el punto de vista de sus normas de edificación.

\section{AGRADECIMIENTOS}

Los autores de este artículo desean agradecer a CONICYT (ANID) por el apoyo financiero derivado de la ejecución del Proyecto Fondef it16i10003, denominado Propuesta de Estándares en infraestructura crítica para edificaciones y viviendas localizadas en zonas de interfaz urbanoforestal, con el objetivo de minimizar el nivel de peligro en la propagación del fuego, modelo a aplicar en la comuna de San José de Maipo. Gracias a este apoyo se pudieron financiar los levantamientos de información en campo, la validación de resultados y entrevistas con habitantes y autoridades de esta comuna.

\section{REFERENCIAS}

Bowman, D., Moreira-Muñoz, A., Kolden, C.A., Chávez, R.O., Muñoz, A.A., Salinas, F., González-Reyes, Á., Rocco, R., de la Barrera, F., Williamson, G.J., Borchers, N., Cifuentes, L.A., Abatzoglou, J.T. \& Johnston, F.H. (2019). Human-environmental drivers and impacts of the globally extreme 2017 Chilean fires. Ambio, 48(4), 350-362. https://doi.org/10.1007/s13280-018-1084-1

Castillo, M.E., Saavedera, J. \& Brull, J. (2019). Severidad del fuego en los mega incendios forestales ocurridos en chile, en 2017. Acciones para mejorar el sistema de protección. Territorium, 26(1), 5-18. https://doi.org/10.14195/1647-7723 26-1 1

Castillo, M.E., Molina, J-R., Rodríguez y Silva, F., García-Chevesich, P.A. \& Garfias, R.A. (2016). A system to evaluate fire impacts from simulated fire behavior in Mediterranean areas of Central Chile. Science of the Total Environment, 579, 1410-1418. http://dx.doi.org/10.1016/i.scitotenv.2016.11.139

Castillo, M.E. (2015). Diagnosis of Forest Fires in Chile. En Bento-Gonçalves, A. \& Vieira, A. (Eds.), Wildland Fires - A worldwide reality (pp.211-224). New York: Nova Science Publishers.

Castillo, M.E., Rodríguez \& Silva, F. (2015a). Quantitative analysis of forest fire extinction efficiency. Forest Systems, 24(2), e032. http://dx.doi.org/10.5424/fs/2015242-06644

Castillo, M.E., Rodríguez y Silva, F. (2015b). Determining response times for the deployment of terrestrial resources for fighting forest fires. A case study: Mediterranean - Chile. Ciencia e Investigación Agraria, 42(1), 97-107. http://dx.doi.org/10.4067/So718-16202015000100010

Castillo, M.E., Julio, G.H. \& Garfias, R.A. (2014). Current status of risk and prognosis of forest fires in Chile. Progress and future challenges. En Paton D. \& Shroder, J.F. (Eds.), Wildfire Hazards, Risks and Disasters (pp. 59-75). Amsterdam: Elsevier. https://doi.org/10.1016/B978-0-12-410434-1.00004-X

Castillo, M.E., Molina, J-R., Rodríguez y Silva, F. \& Julio, G.H. (2013). Fire vulnerability model in Mediterranean ecosystems of South America. Ecological Informatics, 13, 106-113. https://doi. org/10.1016/j.ecoinf.2012.06.004

Castillo, M.E. (2013). Integración de variables y criterios territoriales para la protección contra incendios forestales. Área de estudio: Valparaíso, Chile Central. Tesis Doctoral. Córdoba, España: Universidad de Córdoba.

Castillo, M.E., Garfias, R.A., Julio, G.H. \& González, L.A. (2012). Análisis de grandes incendios forestales en la vegetación nativa de Chile. Interciencia: Revista de ciencia y tecnología de América, 37(11), 796-804.

Castillo, M.E. (2006). El cambio del paisaje vegetal afectado por incendios en la Zona Mediterránea Costera de la Quinta Región de Chile. Tesis Magíster. Santiago de Chile: Universidad de Chile.

Castillo M.E. (1998). Método de Validación para el Simulador de Expansión de Incendios Forestales del Sistema KITRAL. Tesis Pregrado. Santiago de Chile: Facultad de Ciencias Forestales, Universidad de Chile.

Corporación Nacional Forestal CONAF. (2016). Determinación de zonas vulnerables a sufrir daños en incendios forestales y propuestas básicas de prevención en la Comuna de San José de Maipo. Santiago de Chile: CONAF. [Documento de trabajo interno] 
Corporación Nacional Forestal CONAF. (2015). ¿Cómo preparo mi casa y entorno frente a los incendios forestales? Manual de Silvicultura Preventiva. Santiago de Chile: CONAF.

Costafreda-Aumedes, S., Comas, C. \& Vega-García, C. (2017). Human-caused fire occurrence modelling in perspective: a review. International Journal of Wildland Fire, 26, 983-998. https://doi.org/10.1071/ $\underline{W} 17026$

De Luis, M., Baeza, M.J., Raventos, J. \& Hidalgo, J.C. (2004). Fuel characteristics and fire behaviour in mature Mediterranean gorse shrublands. International Journal of Wildland Fire, 13, 79-87. https:// doi.org/10.1071/WF03005

Julio, G.H., Castillo, E. \& Pedernera, P.G. (1995a). Modelación de Combustibles. En Actas del Taller Internacional sobre Prognosis y Gestión en Incendios Forestales (pp.111-128). Santiago de Chile: Universidad de Chile, INTEC-Chile \& INFOR.

Julio, G.H., Pedernera, P.G. \& Castillo, E. (1995b). Diseño Funcional de un Simulador de Incendios Forestales. En Actas de Taller Internacional sobre Prognosis y Gestión en Control de Incendios Forestales (pp.218-226). Santiago de Chile: Universidad de Chile, INTEC-Chile \& INFOR. 